\title{
石蒜碱的全合成及结构修饰研究进展
}

\author{
刘采杰 ${ }^{a}$ 丁泽洋 ${ }^{c}$ 谭 冲 ${ }^{a}$ 袁洪亮 ${ }^{b}$ 季宇涁 $*, a, b$ \\ $\left({ }^{a}\right.$ 哈尔滨商业大学商业工程研究院 哈尔滨 150076) \\ $\left({ }^{b}\right.$ 哈尔滨商业大学生命科学与环境科学研究中心 哈尔滨 150076) \\ ( ${ }^{c}$ 吉林大学化学学院 长春 130000)
}

\begin{abstract}
摘要 石蒜碱是石蒜科生物碱中非常重要的一类异喹啉生物碱衍生物, 近年来药物及有机化学工作者发现石蒜碱及其 衍生物具有抗肿瘤、抗炎、抑制乙酰胆碱酷酶、抗疮疾、保护心血管等多种作用，由于其复杂而独特的分子结构和广 泛的药理活性使得科研工作者们进行了大量的全合成尝试及结构修饰等研究. 作者对石蒜碱的全合成及其结构修饰进 行了综述.
\end{abstract}

关键词 石蒜碱; 全合成; 衍生物; 结构修饰

\section{Progresses in the Total Synthesis and Structure Modification Studies of Lycorine}

\author{
Liu, Yingjie $^{a} \quad$ Ding, Zeyang ${ }^{c} \quad$ Tan, Chong $^{a} \quad$ Yuan, Hongliang $^{b} \quad$ Ji, Yubin $^{*, a, b}$ \\ ( ${ }^{a}$ Business Engineering Research Institute, Harbin University of Commerce, Harbin 150076) \\ ( ${ }^{b}$ Research Center on Life Science and Enviromental Science, Harbin University of Commerce, Harbin 150076) \\ $\left({ }^{c}\right.$ College of Chemistry, Jilin University, Changchun 130000)
}

\begin{abstract}
Lycorine is a very important class of isoquinoline alkaloids of the amaryllidaceae alkaloids. In recent years, pharmaceutical and organic chemists discovered that lycorine and their derivatives have anti-tumor, anti-inflammatory, inhibiting acetylcholinesterase, anti-malarial, cardiovascular protection and other effects. Because of its complex and unique molecular structure and extensive pharmaceutical activities, lycorine has attracted considerable research effort. The recent progresses in the synthesis and structural modification of lycorine are summarized in this review.

Keywords lycorine; total synthesis; derivatives; structure modification
\end{abstract}

中药石蒜为石蒜科 (Amaryllidaceae) 植物石蒜 (Lycoris radiate Herb.)的鳞茎, 全世界共有 20 种石蒜属, 另外还有一些杂交种. 我国拥有 15 个野生品种, 占全属 的四分之三, 在资源上有绝对优势 ${ }^{[1]}$. 石蒜属植物很早 就被用作药用植物来治病疗伤, 作为一种传统的药用植 物, 在临床上有着悠久的应用历史. 石蒜始载于 《图经 本草》, 主要用于 “敷贴肿毒” ; 《本草纲目拾遗》记载 本品可以: “治喉风、痰核、白火丹、肺痛” .

1877 年, 科研人员首次从石蒜科植物洋水仙中分 离出了石蒜碱 ${ }^{[2]}$, 但其结构直到 1935 年才得以确立 ${ }^{[3]}$. 1959 年, 科学家进一步通过单晶确定了石蒜碱的立体
结构 ${ }^{[4]}$.

石蒜碱属于异喹啉类生物碱, 为吡咯并菲啶的衍生 物. 吡咯并菲啶天然生物碱结构如图 1. 现代药理学研 究证明, 石蒜碱具有抗炎、抑制乙酰胆碱酯酶、抗症疾、 保护心血管以及诱导肿瘤细胞调亡等多种作用, 石蒜碱 还具有广泛的抗病毒活性，如 SARS 病毒、疮疹病毒、 牛痘病毒、蓬塔托罗病毒、裂谷热病毒等 ${ }^{[5 \sim 15]}$. 另一方 面, 对石蒜碱及其衍生物的研究, 也极大地推动了人们 在治疗肿瘤、老年痴呆、病毒感染等严重危害人类健康 的疾病方面的进展 ${ }^{[16 ~ 18]}$. 因此, 对于石蒜碱的全合成及 石蒜碱的结构修饰以寻求活性更强的石蒜碱衍生物, 对

*E-mail: lyj850527@163.com

Received September 24, 2014; revised October 29, 2014; published online December 10, 2014

Project supported by the Harbin University of Commerce Doctoral Research Project (No. 14LG12) and the Natural Science Fund for Young Teachers of Harbin University of Commerce (No. HCUL2013019).

哈尔滨商业大学博士科研启动项目(No. 14LG12)、哈尔滨商业大学青年教师自然科学基金(No. HCUL2013019)资助项目 
于药物合成以及新药研发具有重要意义.

\section{1 石蒜碱的全合成研究概述}

石蒜碱(Lycorine)的全合成一般是构筑如图 1 所示 的 A、B、C、D 环, 通过文献调研发现, 石蒜碱的全合 成一般是按照以下五种成环顺序: (1) $\mathrm{AD} \rightarrow \mathrm{ADB} \rightarrow$ $\mathrm{ADBC}$ 环骨架的建立; (2) $\mathrm{AD} \rightarrow \mathrm{ADC} \rightarrow \mathrm{ADCB}$ 环骨架的 建立; (3) $\mathrm{DC} \rightarrow \mathrm{DCA} \rightarrow \mathrm{DCAB}$ 环骨架的建立; (4) $\mathrm{A} \rightarrow \mathrm{ACD} \rightarrow \mathrm{ACDB}$ 环骨架的建立; (5) A $\rightarrow \mathrm{AC} \rightarrow \mathrm{ACDB}$ 环 骨架的建立. 直接全合成石蒜碱的文献相对较少, 而全

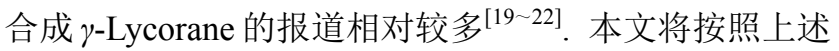
五种顺序对这类生物碱的全合成进行简要介绍.

\section{$1.1 A D \rightarrow A D B \rightarrow A D B C$ 环骨架的建立}

早在 1975 年, Murata 等 ${ }^{[23]}$ 从外消旋氨酯一酯 1 出发 (Scheme 1), 按照构建 $\mathrm{AD} \rightarrow \mathrm{ADB} \rightarrow \mathrm{ADBC}$ 环骨架的顺 序, 经多步合成了石蒜碱. 从外消旋氨酯-酯经氧化、还 原等多步合成关键中间体产物 $\mathbf{5}$, 通常被叫作 Trossell 产物, 利用此化合物可以顺利地得到目标产物石蒜<smiles>OC1C=C2C=CN3Cc4cc5c(cc4C(C1O)C23)OCO5</smiles>

Lycorine<smiles></smiles>

Zephyranthine<smiles>c1c2c(cc3c1OCO3)[C@H]1CCC[C@@H]3CCN(C2)[C@H]31</smiles>

$\gamma$-Lycorane<smiles>O[C@H]1CC=C2CCN3Cc4cc5c(cc4[C@@H]2[C@@H]13)OCO5</smiles>

图 1 吡咯并菲啶天然生物碱

Figure 1 Pyrrolo[de]phenanthridine natural alkaloid

碱 ${ }^{[24]}$. 其中, 用间氯过氧苯酸氧化重排 Trossell 产物得 到差向异构的环氧化合物的混合物, 然后与二苯基二硒 醚作用得到三种混合物. 化合物 7a 的分离产率为 33\%. 很多合成工作者都以此作为目标产物逆合成分析来设 计反应物，所合成的化合物 $\mathbf{5}$, 经历氧化、还原、酰化等

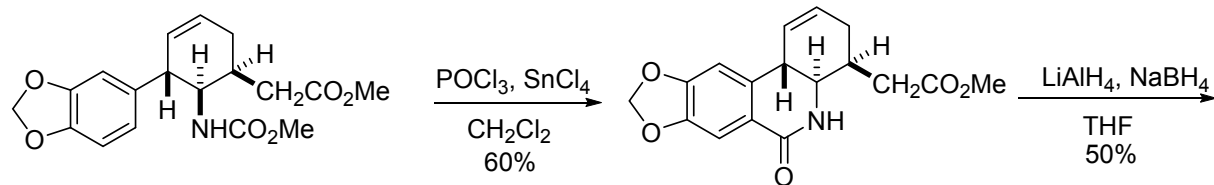

1

2<smiles>O=C(O)C[C@H]1CC=CC2c3cc4c(cc3C(=O)N[C@H]21)OCO4</smiles>

3

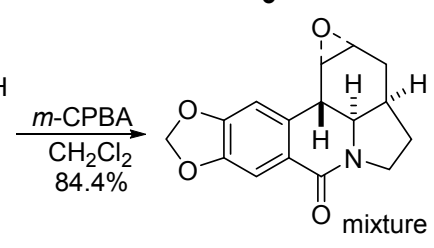

6<smiles>O=C1c2cc3c4cc2C(C=CC[C@H]2CCN1C32)OCO4</smiles>

5

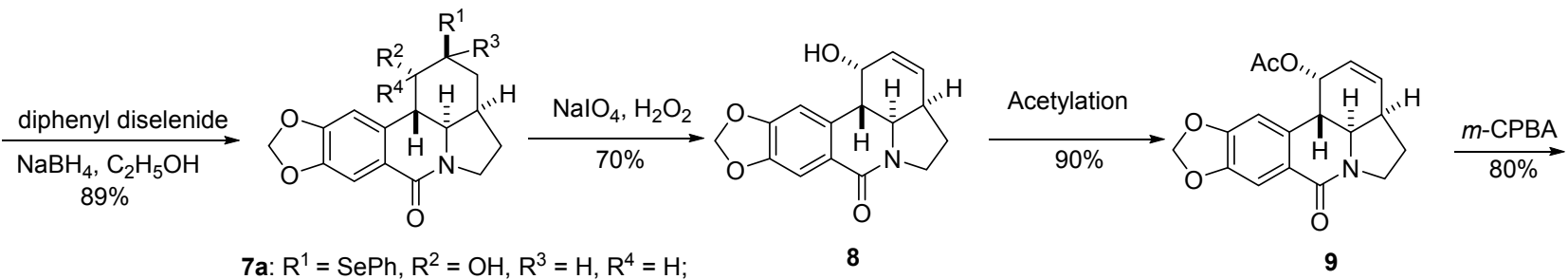

7a: $\mathrm{R}^{1}=\mathrm{SePh}, \mathrm{R}^{2}=\mathrm{OH}, \mathrm{R}^{3}=\mathrm{H}, \mathrm{R}^{4}=\mathrm{H}$;

7b: $\mathrm{R}^{1}=\mathrm{OH}, \mathrm{R}^{2}=\mathrm{SePh}, \mathrm{R}^{3}=\mathrm{H}, \mathrm{R}^{4}=\mathrm{H}$;

7c: $R^{1}=\operatorname{SePh}, \mathrm{R}^{2}=\mathrm{H}, \mathrm{R}^{3}=\mathrm{H}, \mathrm{R}^{4}=\mathrm{OH}$;

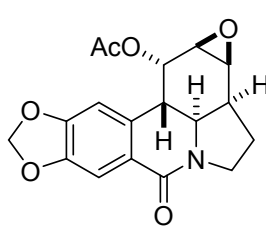

10<smiles>O=C1c2cc3c(cc2[C@H]2[C@@H]4[C@@H](CCN14)CC(O)[C@@H]2O)OCO3</smiles>

11

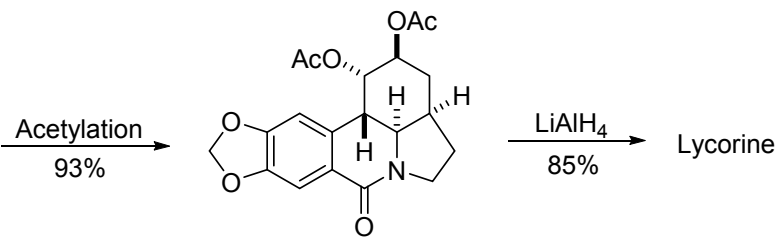

12

图式 1 Murata 课题组报道的石蒜碱的全合成

Scheme 1 Total synthesis of lycorine reported by Murata 
多步反应合成了石蒜碱，下文通过 Trossell 产物合成石 蒜碱的合成路线不再重复赘述.

1984 年, Umezawa 课题组 ${ }^{[25]}$ 利用原料易得的反应 物通过 Diels-Alder 反应和鲁宾逊成环反应制备化合物 13 和 23, 中间产物 19 的成功合成, 为最终实现石赫碱 的全合成奠定了坚实的基础. 整个路线虽然步骤略多, 但产率较高. 该工作同样是按照构建 $\mathrm{AD} \rightarrow \mathrm{ADB} \rightarrow$ ADBC 环骨架的顺序合成的石赫碱. 首先介绍第一种方 法: 利用市售的硝基苯乙烯和丁二烯发生 Diels-Alder 反 应(Scheme 2), 得到反应物 13, 在 $m$-CPBA 的条件下氧 化得到环氧化合物的两种异构体 14, 其 $\alpha: \beta$ 构型比为
1：1, 然后再通过还原、酰化、再氧化得到化合物 $\mathbf{1 8}$, 发 生的 Pictet-Spengier 反应成功地构建了 B 环. 所合成的 中间产物 19 再多步反应得到 Torssell 产物而进一步合成 石蒜碱.

第二种获得中间产物 19 的方法见 Scheme 3, 利用 反应物 22 和甲基乙烯基酮发生鲁宾逊成环反应合成化 合物 23, 然后通过消除酯化、以及 Curtius rearrangement 多步反应得到化合物 25, 同样经过 Pictet-Spengier 反应 构筑 B 环得到中间产物 19, 最后也是通过 Trossell 产物 来合成石蒜碱 ${ }^{[25]}$.

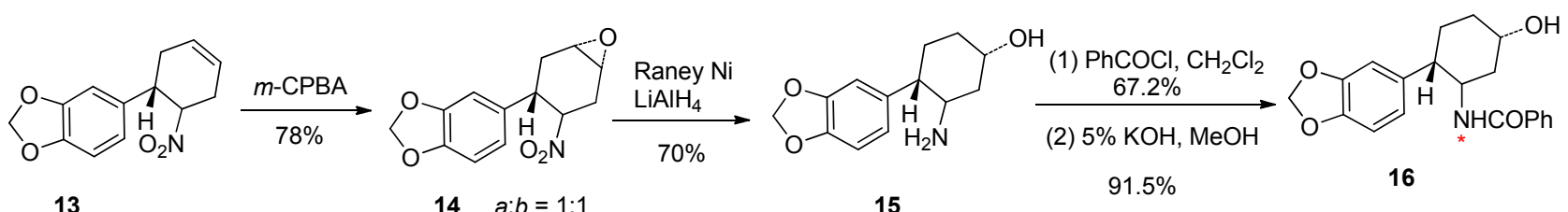

13

$14 a: b=1: 1$

15<smiles>CNC1CC(=O)CCC1(c1ccccc1)c1ccc2c(c1)OCO2</smiles>

17

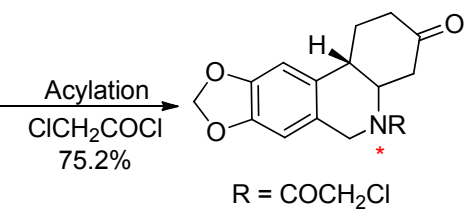

20

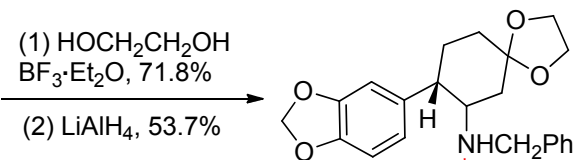

$18^{*}$

(1) $t$ - $\mathrm{BuOk}, t-\mathrm{BuOH}, 32 \%$ (2) $\mathrm{Me}_{2} \mathrm{NHHCl}_{2}, \mathrm{NaBH}_{3} \mathrm{CN}$, $\mathrm{MeOH}, 43 \%$

(3) $m$-CPBA, $95 \%$

(4) under $\mathrm{Ar}, 200^{\circ} \mathrm{C}, 73.1 \%$<smiles></smiles>

21

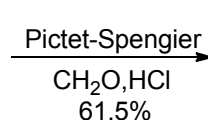
$61.5 \%$

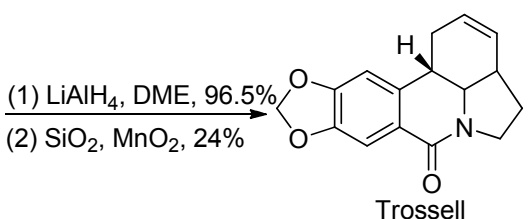

图式 2 Umezawa 及合作者报道的中间产物 19 的合成方法一

Scheme 2 A method of synthesis intermediate 19 reported by Umezawa and co-workers

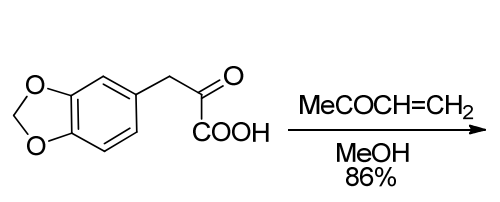

22<smiles>O=C1CCC(c2ccc3c(c2)OCO3)C(O)(C(=O)O)C1</smiles>

23
(1) $\mathrm{Zn}, \mathrm{AcOH}, 91.4 \%$ (2) $\mathrm{H}_{2} \mathrm{SO}_{4}, \mathrm{MeOH}, 96.1 \%$

(3) $\mathrm{HOCH}_{2} \mathrm{CH}_{2} \mathrm{OH}, 98.1 \%$ (4) $\mathrm{NaOMe}, \mathrm{MeOH}, 81.8 \%$<smiles>COC(=O)C1CC2(CC[C@H]1c1ccc3c(c1)OCO3)OCCO2</smiles>

24

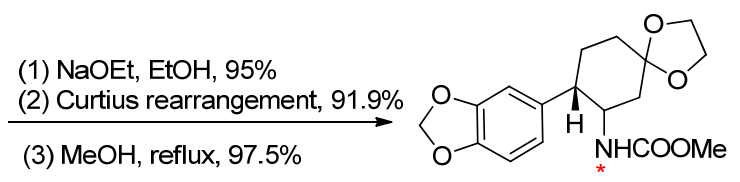

25

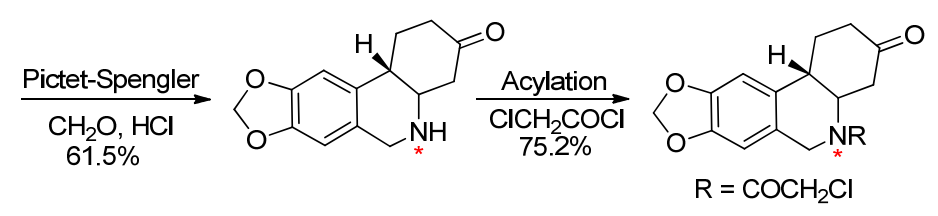

19

20

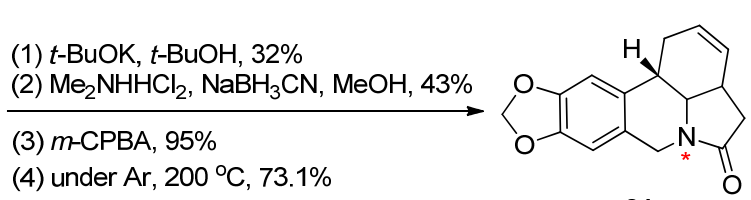

21
(1) $\mathrm{LiAlH}_{4}$, DME, 96.5\% (2) $\mathrm{SiO}_{2}, \mathrm{MnO}_{2}, 24 \%$<smiles>O=C1c2cc3c(cc2[C@H]2CC=CC4CCN1C42)OCO3</smiles>

图式 3 Umezawa 及合作者报道的中间产物 19 的合成方法二

Scheme 3 Another method of synthesis intermediate 19 reported by Umezawa and co-workers 


\section{$1.2 A D \rightarrow A D C \rightarrow A D C B$ 环骨架的建立}

1978 年, Torssell 等 ${ }^{[26]}$ 最早利用硝基烯化合物 26 和 双烯 27 发生 Diels-Alder 反应生成化合物 28, 该反应的 发生实际上同时生成了化合物 28 的异构体, 但是在反 应的后处理上利用重结晶的方法即可将二者分离开, 得 到单一的化合物 28. 然后先经过还原硝基构建 $\mathrm{C}$ 环, 再 还原羰基双键得到化合物 29, 在三氯氧磷催化下与氯 甲酸乙酯反应得到 Torssell 产物, 即是按照构建 $\mathrm{AD} \rightarrow \mathrm{ADC} \rightarrow \mathrm{ADCB}$ 环骨架的顺序合成的石蒜碱, 相对 Umezawa 的工作产率相对较低(Scheme 4).

2009 年, Tomioka 课题组 ${ }^{[27]}$ 利用手性配体控制催化 芳基锂化合物 30 对含有两个 $\alpha, \beta$-不饱和羰基部分的迈 克尔受体 31 发生不对称串联共轭加成反应(Scheme 5), 对映选择性地合成中间产物化合物 32, 进一步对映选 择性地发生分子内的迈克尔加成得到化合物 33, 同时 获得三个手性中心, 然后经历 Curtius 重排和 Bischler-

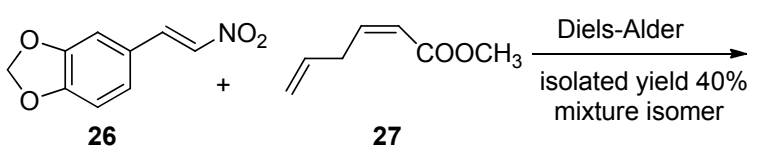<smiles>COC(=O)[C@H]1C=CC[C@H](c2ccc3c(c2)OCO3)[C@H]1[N+](=O)[O-]</smiles>

28<smiles>O=C1c2cc3c4cc2[C@@H](CC=CC2CCN1C32)OCO4</smiles>

29

图式 4 Torssell 课题组按照构建 $\mathrm{AD} \rightarrow \mathrm{ADC} \rightarrow \mathrm{ADCB}$ 环骨架 的顺序合成的石蒜碱

Scheme 4 Synthesis of lycorine according to the building $\mathrm{AD} \rightarrow \mathrm{ADC} \rightarrow \mathrm{ADCB}$ ring skeleton by Torssell group<smiles>COC(=O)CC=CCC1(CC=CCOC(C)(C)C)OCCO1</smiles>

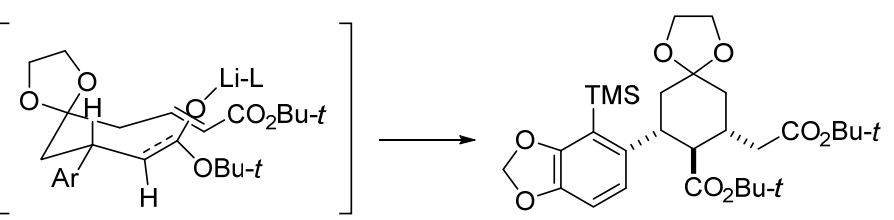

32

33

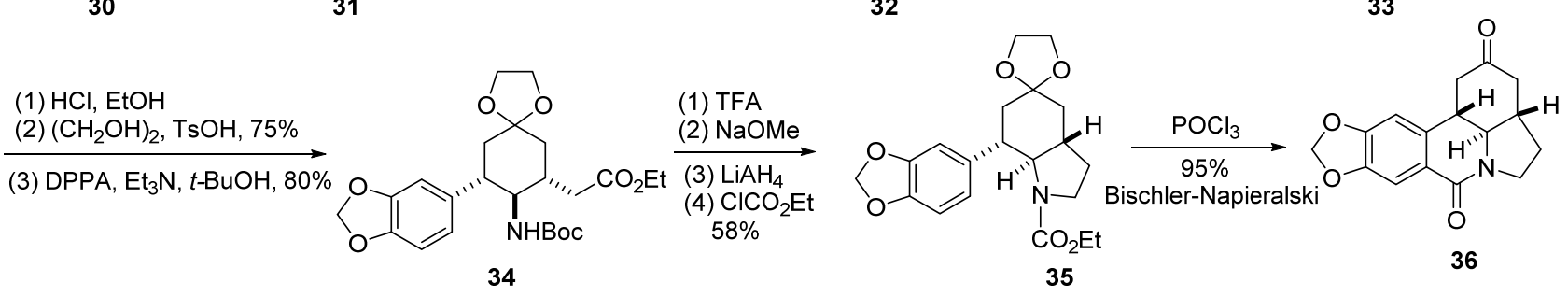

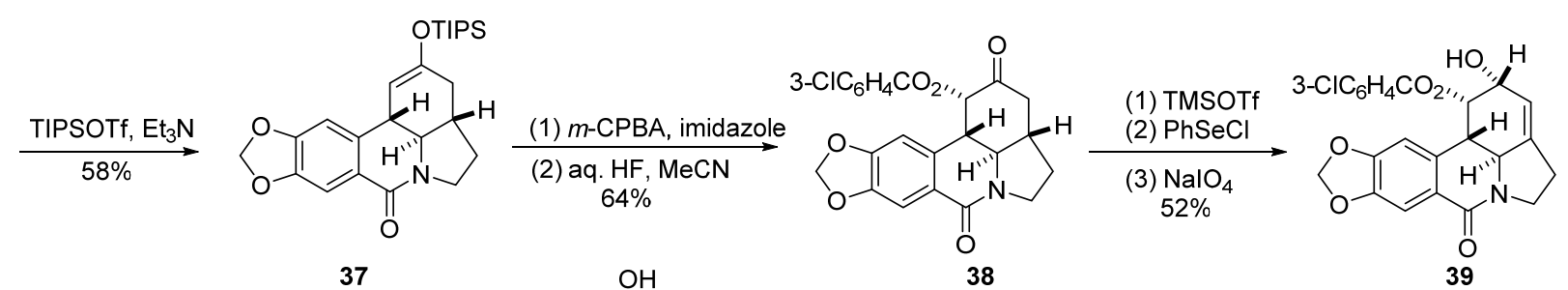

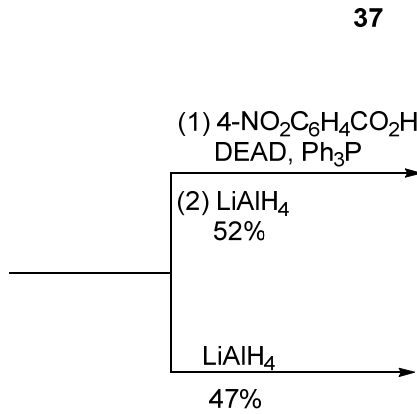<smiles>O[C@H]1C=C2CCN3Cc4cc5c(cc4[C@@H]([C@H]1O)[C@H]23)OCO5</smiles><smiles>O[C@H]1C=C2CCN3Cc4cc5c(cc4[C@@]2(CCN3)[C@H]1O)OCO5</smiles>

2-epi-lycorine

图式 5 Tomioka 课题组利用不对称方法参与合成石蒜碱

Scheme 5 Synthesis of lycorine with asymmetric method reported by Tomioka group 
Napieralski 反应合成具有石蒜碱骨架的化合物 36, 利用 化合物 36 合成 TIPS 烯醇 37, 化合物 37 经过 Magnus 反应过程立体专一的得到化合物 $\mathbf{3 8}^{[28]}$, 为石蒜碱 $C(1)$ 位羟基的立体化学的建立奠定基础. 该方法是按照构建 $\mathrm{AD} \rightarrow \mathrm{ADC} \rightarrow \mathrm{ADCB}$ 环骨架的顺序合成的石赫碱. 该工 作的突出亮点是利用手性配体控制两个 $\mathrm{C}-\mathrm{C}$ 键和三个 手性中心的形成, 同时手性配体 $\mathbf{L}$ 可以回收重复利用, 该工作是第二例不对称合成方法参与合成的石蒜碱，为 其它生物碱的合成开辟了新的方法, 具有重要的意 义.

2014 年, 邵课题组 ${ }^{[29]}$ 全合成制备了石蒜碱, 该反应 的顺利进行是制备化合物 48 (Scheme 6), 首先利用非常 简单的反应物乙烯基溴发生 Sonogashira 反应以及 Henry 反应得到化合物 41, 然后在手型配体控制催化下 使其与丙二酸二叔丁酯发生 1,4 加成反应得到化合物 42, 以 $91 \%$ 的产率得到化合物 $\mathbf{4 2}$ 并且得到 $93 \%$ 高 $e e$ 值,
该工作是第三例涉及了不对称合成，该工作与之前工作 的不同点在于：手型配体控制高区域选择性的合成了化 合物 42, 此化合物并没有石蒜碱的任何骨架，然后经过 酸化、酯化得到化合物 44, 然后化合物 44 经历非对映 选择性的分子内的迈克尔加成反应环化得到非对映异 构体，核磁显示其 $d . r$. 比率为 $6: 1$, 柱层析后以 $60 \%$ 的 高产率分离出化合物 $\mathbf{4 5}$, 再通过还原, 环化等反应可以 制备具备石蒜碱骨架化合物 50, 最终利用化合物 50 制 备目标产物石蒜碱. 该方法也是按照构建 $\mathrm{AD} \rightarrow \mathrm{ADC} \rightarrow \mathrm{ADCB}$ 环骨架的顺序合成的石蒜碱.

\section{$1.3 \mathrm{DC} \rightarrow \mathrm{DCA} \rightarrow \mathrm{DCAB}$ 环骨架的建立}

1996 年, Smyth 课题组 ${ }^{[30]}$ 首次不对称全合成了石蒜 碱,该反应首先利用手性的苯甲酰胺 54 和 2-溴乙基乙酸 酯 53 经奇还原烷基化、酯皇化反应引入氮原子建立 $\mathrm{C}$ 环，然后与溴代芳基酰氯反应构建 A、B 环，共经历 15

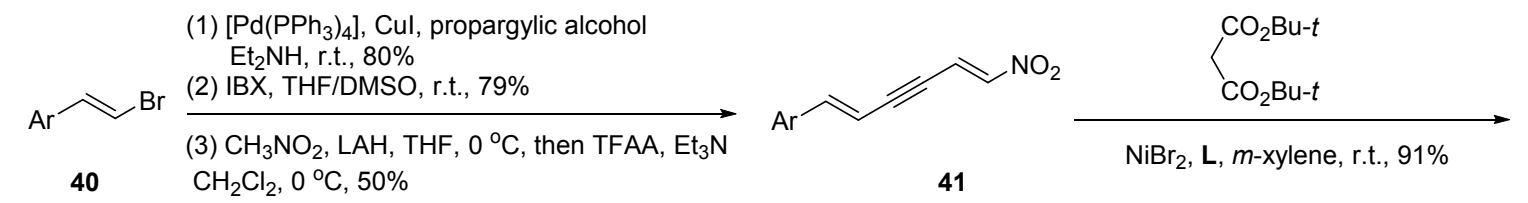<smiles>CCOC(=O)C[C@H]1CC(=O)C[C@@H]([Al])[C@H]1[N+](=O)[O-]</smiles>

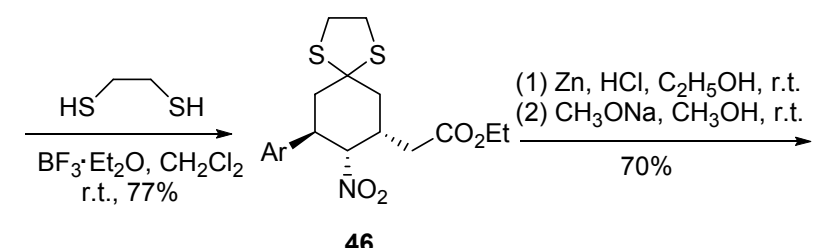<smiles></smiles>

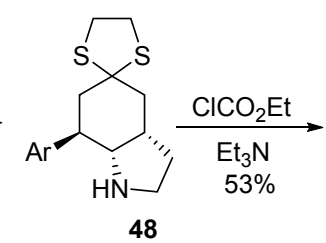

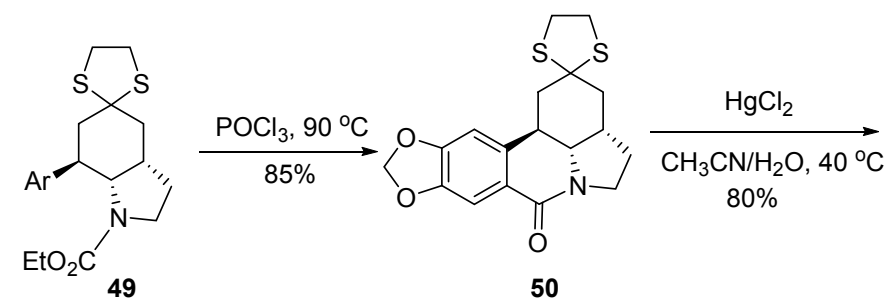

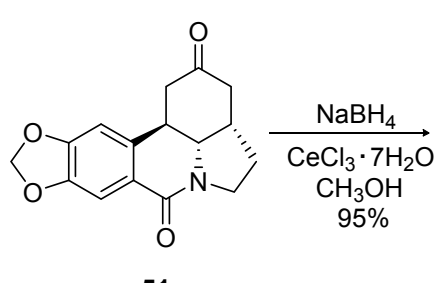<smiles>O=C1c2cc3c(cc2[C@@H]2CC(O)C[C@@H]4CCN1[C@@H]42)OCO3</smiles>
50 51 52

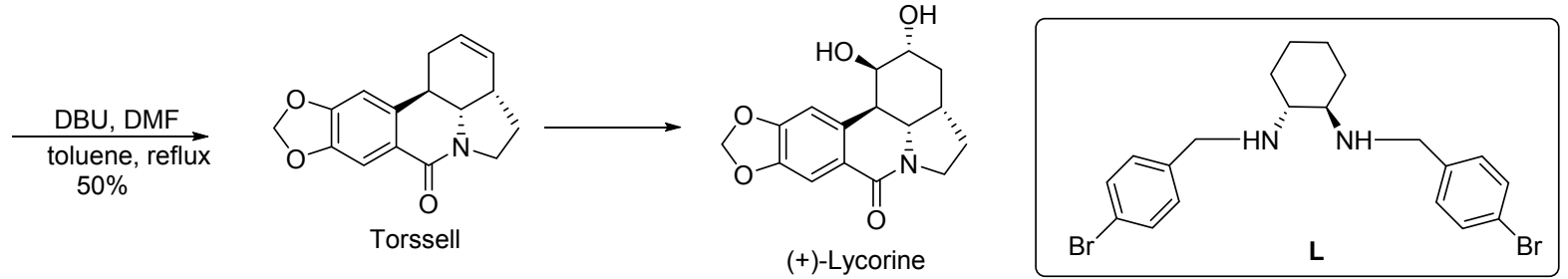

图式 6 邵课题组按照构建 $\mathrm{AD} \rightarrow \mathrm{ADC} \rightarrow \mathrm{ADCB}$ 环骨架的顺序全合成制备了石蒜碱

Scheme 6 Synthesis of lycorine according to the building $\mathrm{AD} \rightarrow \mathrm{ADC} \rightarrow \mathrm{ADCB}$ ring skeleton by Shao group 
步合成了历伯石蒜碱(Scheme 7), 此方法最关键的是合 成中间体化合物 61, 然后按照 Trossell 方法合成目标产 物石蒜碱, 该工作的亮点是利用手性反应物采用伯奇还 原烷基化反应新合成策略全合成石蒜碱, 反应虽然步骤 略多, 但是产率相对较高, 为其他天然产物的全合成提 供了方法指导, 具有重要的意义.

\section{$1.4 \mathrm{~A} \rightarrow \mathrm{ACD} \rightarrow \mathrm{ACDB}$ 环骨架的建立}

1981 年, Hecht 等 ${ }^{[31]}$ 首先利用胡椒醛与对甲氧基苯
胺和噻吩二氧化物反应得到化合物 65 (Scheme 8),低温 下可以分离化合物 66, 利用酰胺作为亲二烯体发生分 子内 $[4+2]$ 环加成反应顺利得到化合物 67 , 然后经还 原、环化得到 Trossell 产物, 从而进一步合成了石赫碱. 该工作全合成石蒜碱是按照 $\mathrm{A} \rightarrow \mathrm{ACD} \rightarrow \mathrm{ACDB}$ 环骨架 的形成顺序, 其中 C、D 环因发生分子内 $[4+2]$ 环加成反 应同时形成.

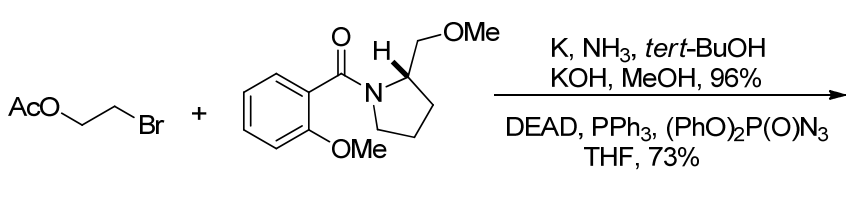

53

54<smiles>COCC1CCCN1C(=O)c1ccccc1OC</smiles>

55<smiles>COCN1CCCN1C(=O)C1(CN)C=CCCC1=O</smiles>

56

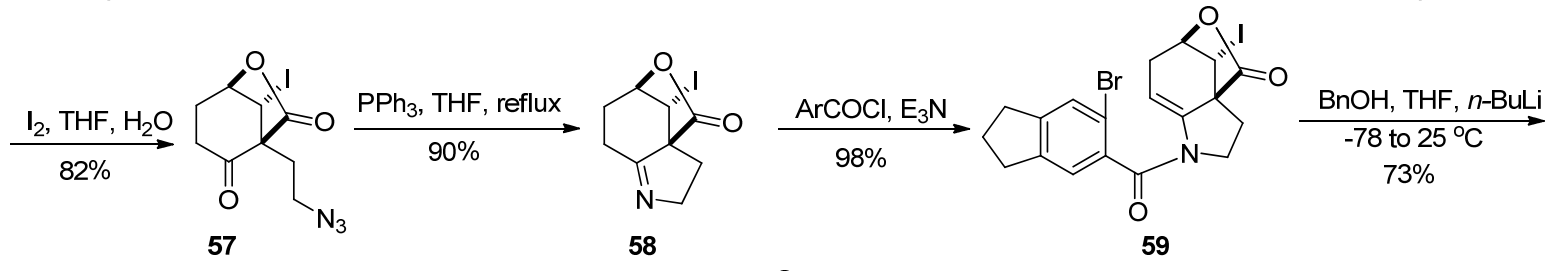

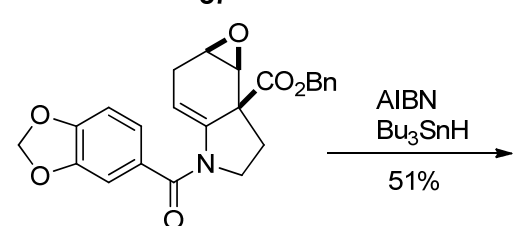

60<smiles></smiles>

61

Similar product 6

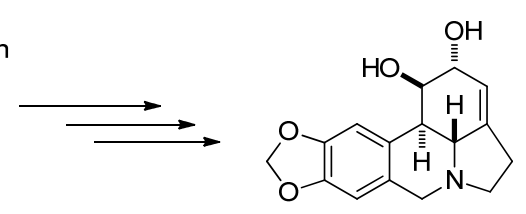

(+)-Lycorine

图式 7 Smyth 及合作者首次不对称全合成了石蒜碱

Scheme 7 Synthesis of lycorine with asymmetric method for the first time by Smyth and co-workers

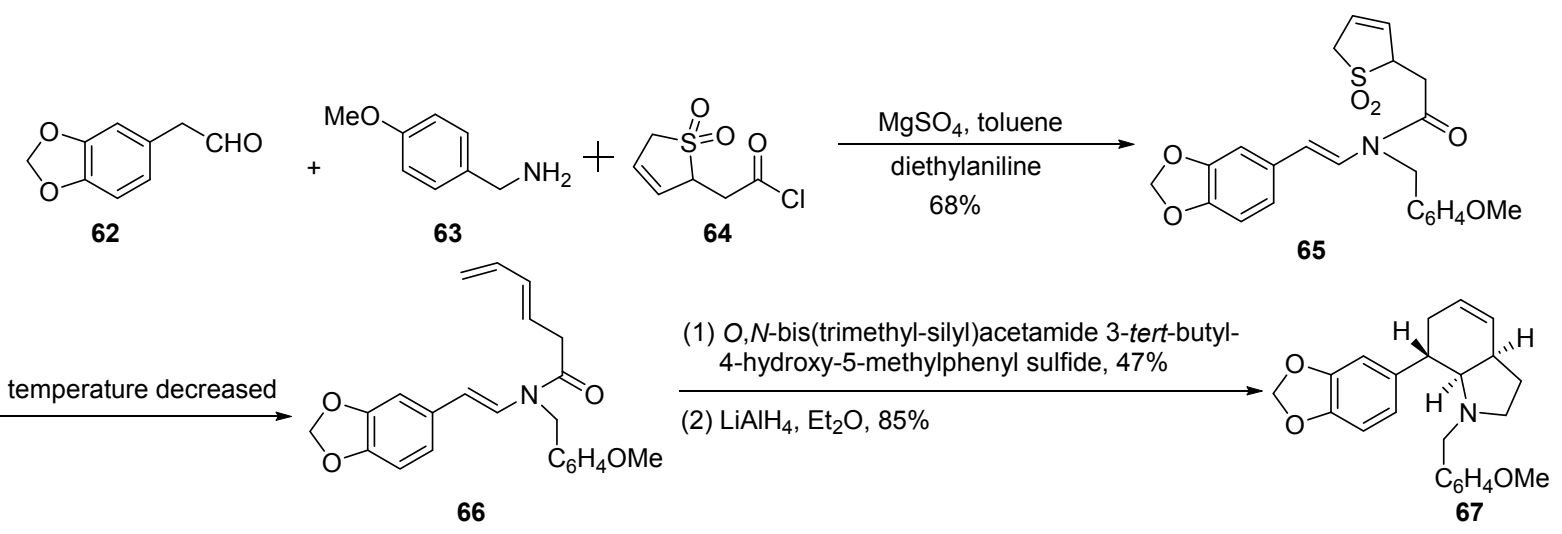

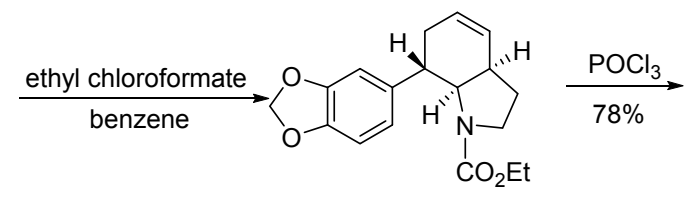

68<smiles>O=C1c2cc3c(cc2C2CC=C[C@H]4CCN1C24)OCO3</smiles>

Torssell

图式 8 Hecht 及合作者按照 $\mathrm{A} \rightarrow \mathrm{ACD} \rightarrow \mathrm{ACDB}$ 环骨架的形成顺序全合成了石蒜碱

Scheme 8 Synthesis of lycorine according the buliding $A \rightarrow A C D \rightarrow A C D B$ ring skeleton by Hecht and co-workers 


\section{$1.5 \mathrm{~A} \rightarrow \mathrm{AC} \rightarrow \mathrm{ACDB}$ 环骨架的建立}

1988 年, Boeckman 课题组 ${ }^{[32]}$ 利用环丙基亚胺离子 重排合成石蒜碱(Scheme 9), 亚胺离子亲核性较弱, 为 了增强反应活性, 使其与活性较强的酸性卤化物 69 反 应可以经多步顺利得到石蒜碱异构体. 而化合物 69 是 利用黄樟素作为反应物得到的, 合成石蒜碱以黄樟素作 为反应物仅需要 13 步, 产率相对较高. 该工作是按照 $\mathrm{A} \rightarrow \mathrm{AC} \rightarrow \mathrm{ACDB}$ 环骨架的顺序, B、D 环同时合成.

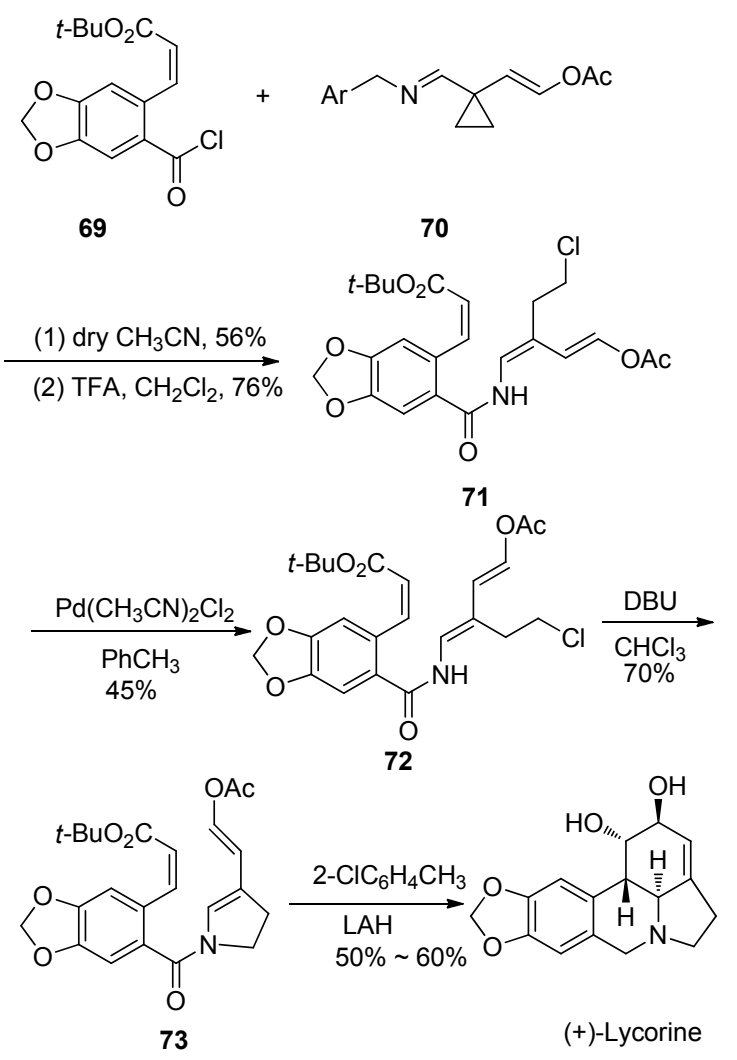

图式 9 Boeckman 课题组是按照 $\mathrm{A} \rightarrow \mathrm{AC} \rightarrow \mathrm{ACDB}$ 环骨架的 顺序全合成石蒜碱

Scheme 9 Synthesis of lycorine according the buliding $\mathrm{A} \rightarrow \mathrm{AC} \rightarrow \mathrm{ACDB}$ ring skeleton by Boeckman group

\section{2 石蒜碱结构修饰研究进展}

近年来, 药物及有机化学工作者在石蒜碱的修饰与 改造方面做了大量的工作. 图 2 列举了几个结构修饰后 的石蒜碱衍生物, 对石蒜碱的修饰主要集中在: (1)对 $\mathrm{C}(1)$ 和 $\mathrm{C}(2$ )位置的着基进行修饰; (2)将 $\mathrm{C}(2)$ 位置的羟基 氧化成羰基; (3) C (7) 位置氧化和 D 环双键的还原; (4)将 $\mathrm{E}$ 环开环和 D 环的芳香化; (5) A 环的开环. 因此, 通过 对石蒜碱类化合物的结构进行改造, 寻找活性更强的石 蒜碱类衍生物的研究工作倍受关注. 本文对石蒜碱修饰 与改造的研究情况进行了综述.

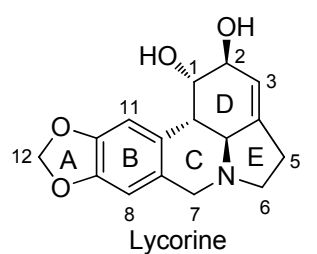<smiles>CC(C)O[C@H]1C(=O)C=C2CCN3Cc4cc5c(cc4[C@@H]1[C@H]23)OCO5</smiles><smiles></smiles><smiles></smiles>

图 2 石蒜碱和石蒜碱衍生物

Figure 2 The structure of lycorine and select derivatives

\section{$2.1 \mathrm{C}(1)$ 和 $\mathrm{C}(2)$ 位置羟基的修饰}

近年来, 科研工作者们大多选择对 C(1)和 C(2)位置 的两个游离羟基进行结构改造(Scheme 10). 最简单的 结构改造, 也是大多数对石蒜碱进行结构改造都经历的 中间体，即将石蒜碱 C(1)和 C(2) 位置的两个游离羟基被 乙酰氧基取代(74), 双乙酰氧基取代也是改变石蒜碱溶 解性的方法, 或是单独其中一个游离羟基被乙酰氧基取 代(75), 双取代的结构改造其抗肿瘤活性并没有明显的 升高，而其 C(1)位置被乙酰氧基取代后对抑制乙酰胆碱 酷酶的活性是加兰他敏的两倍以上 ${ }^{[33]}$.

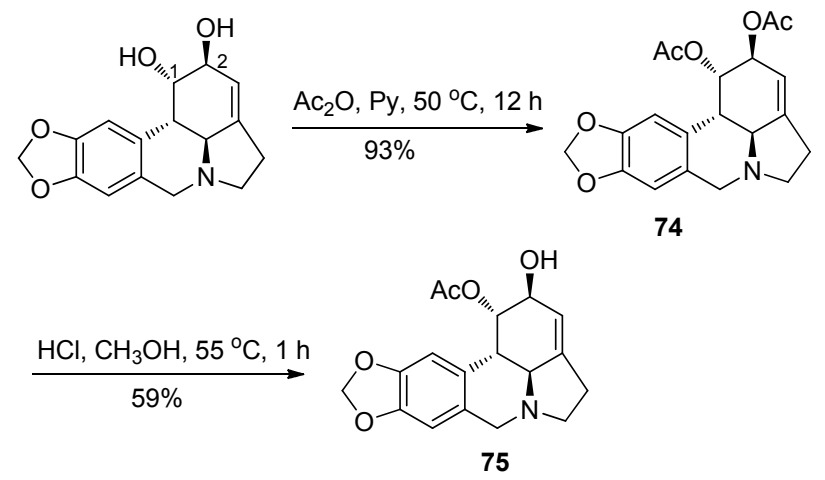

图式 10 石蒜碱 C(1)和 C(2)位置的简单修饰 Scheme 10 Simple modification at $\mathrm{C}(1)$ and $\mathrm{C}(2)$ position of lycorine

2014 年, 尹正课题组 ${ }^{[34]}$ 对石蒜碱进行修饰, 并研究 其抑制登革热病毒活性，探讨其构效关系. 利用市售的 石蒜碱为反应物, 将石蒜碱 C(1)位置羟基乙酰化修饰得 化合物 76, 测得的抑制登革热病毒活性高于石蒜碱的 两倍, 所以保留 $\mathrm{C}(1)$ 位置的乙酰基进一步对 $\mathrm{C}(2)$ 羟基进 行修饰, 使其与酰氯发生酯化作用, 得到一系列修饰后 的产物 77, 但结果并不如所预想的, 其抗病毒活性都大 大降低, 且随着碳链的增长活性降低(Scheme 11). 


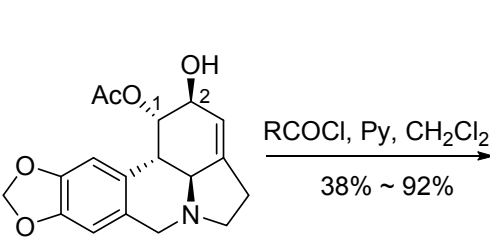

76

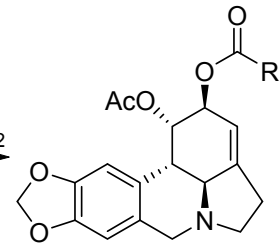

77
$\mathrm{R}=\mathrm{CH}_{2} \mathrm{CH}_{3},\left(\mathrm{CH}_{2}\right)_{3} \mathrm{CH}_{3},\left(\mathrm{CH}_{2}\right)_{4} \mathrm{CH}_{3},\left(\mathrm{CH}_{2}\right)_{3} \mathrm{COOH}$, $\mathrm{CH}_{2} \mathrm{NHBoc}, \mathrm{C}\left(\mathrm{CH}_{3}\right)_{3}$, $\mathrm{Ph}, 4-\mathrm{NO}_{2} \mathrm{C}_{6} \mathrm{H}_{4}, 4-\mathrm{ClC}_{6} \mathrm{H}_{4}$

图式 11 尹正课题组对石蒜碱 C(1) 和 C(2)位置进行酰化修饰 Scheme 11 Acylation modification at C(1) and C(2) position of lycorine by Zheng Yin group

为了考察手型对抗病毒活性的影响, 尹正等 ${ }^{[34]}$ 利 用光延反应使其 $\mathrm{C}(2)$ 位置构型发生翻转, 得到翻转产物 78 和 79, 但结果也不能令人满意, 抗病毒活性仍然大大
降低，但这也不能排除对 $\mathrm{C}(2)$ 位置羟基修饰的干扰. 因 此, 对 C(2)位置的手性研究仍然是未来科研工作者研究 的方向(Scheme 12).

基于生物等排原理，将 $\mathrm{C}(2)$ 位置的羟基变为胺基， 合成了如 Scheme 13 所示的一系列化合物 81 84, 但其 抗病毒活性仍然不如石蒜碱. 因此以上结果显示, $C(2)$ 位羟基是抑制登革热病毒活性的重要基团 ${ }^{[34]}$

2011 年, Rimando 课题组 ${ }^{[35]}$ 研究了石蒜碱衍生物对 水产养殖业的柱形病毒的抑制作用(图 3). 研究表明，对 $\mathrm{C}(1)$ 和 $\mathrm{C}(2)$ 差基双酰基化 87 的抗柱形病的活性比单独 对 $\mathrm{C}(2)$ 着弪基酰基化 86 活性高，而将石蒜碱成盐得到的 化合物 88 虽然改变了溶解性但是抗柱形病毒的活性并 没有提高.<smiles>CC(=O)O[C@H]1c2cc3c(OCO)cc2CN2CCC(=C[C@H]1O)C32</smiles>

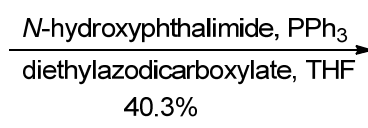
$40.3 \%$

图式 12 尹正课题组利用光延反应将 C(2)位置构型发生翻转<smiles>CO[C@H]1C=C2CCN3Cc4cc5c(cc4[C@@H]([C@H]1O)[C@H]23)OCO5</smiles>

85 $55.4 \% \quad \mathrm{CH}_{3} \mathrm{ONa}, \mathrm{MeOH}$<smiles>[R]c1cn([C@@H]2C=C3CCN4Cc5cc6c(cc5[C@@H]([C@H]3O)[C@H]24)OCO6)nn1</smiles>

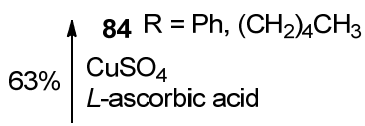<smiles>CC(=O)O[C@H]1[C@@H](O)C=C2CCN3Cc4cc5c(cc4[C@H]2[C@H]13)OCO5</smiles>

76<smiles>CO[C@H](Cl)[C@H]1COc2cc3c(cc21)[C@H]1C2C(=C[C@H]4O[C@@H]24)CN1CC3</smiles>

80

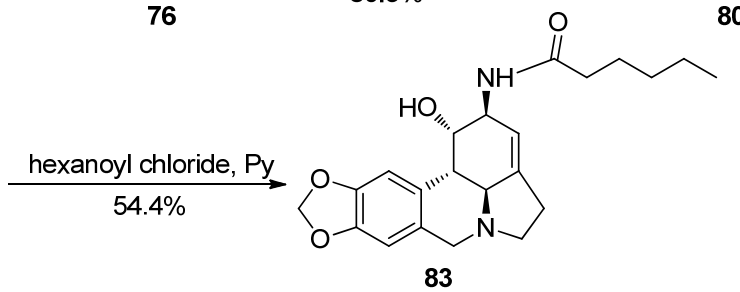<smiles>N[C@H]1C=C2CCN3Cc4cc5c(cc4[C@@H]([C@H]1O)[C@H]23)OCO5</smiles>

81

82<smiles></smiles>

79

图式 13 尹正课题组基于生物电子等排原理将石蒜碱 $\mathrm{C}(2)$ 位置羟基变为胺基

Scheme 13 The hydroxy group at the C(2) position could potentially be converted into an amino group based on bioisosterism by Zheng Yin group 
<smiles></smiles>

86

$\mathrm{R}=2-\mathrm{Me}, 4-\mathrm{Me}, 2-\mathrm{Cl}, 4-\mathrm{Cl}$

$38 \% \sim 82 \%$<smiles></smiles>

87<smiles>[R]c1ccc(C(=O)O[C@H]2C=C3CC[N+]4(CC)Cc5cc6c(cc5[C@]3(CC2)[C@@H]4OC(=O)c2cccc([R])c2)OCO6)cc1</smiles>

88

图 3 Rimando 课题组报道的石蒜碱衍生物的结构

Figure 3 Structures of lycorine analogues reported by Rimando group

2011 年, Kornienko 课题组 ${ }^{[36]}$ 选取从石蒜科植物中 提取的石蒜碱, 主要将石蒜碱 C(1)位置的差基进行酰 化、醚化修饰, 合成了 32 个石蒜碱衍生物, 并对所修饰 的产物进行体外抗肿瘤活性测试, 选取八种癌症细胞株 进行体外篮选, 都能得到良好的抗肿瘤活性(图 4). 其 中, 在强碱 $\mathrm{NaH}$ 的条件下, 利用烯丙基溴和石蒜碱反应 的生成化合物 97, 对神经胶质瘤细胞的抑制活性比石 蒜碱的活性强 100 倍.

2014 年, Kornienko 课题组 ${ }^{[37]}$ 基于以上的工作基础, 选取卤代烃和石蒜碱发生醚化反应. 在强碱 $\mathrm{NaH}$ 的条
件下，石蒜碱并没有发生季铵化反应，而是卤代烃与石 蒜碱 $\mathrm{C}(1)$ 和 $\mathrm{C}(2)$ 位置的羟基发生双醚化反应或是单醚 化反应，合成 35 个石蒜碱衍生物，能够以 $14 \% \sim 90 \%$ 的 产率得到目标化合物. 图 5 列举化合物 98 109, 并对 修饰产物进行体外抗肿瘤活性测试, 选取六种癌症细胞 株, 虽然不能全部达到良好的抗肿瘤活性, 但是为抗癌 药物的研究奠定了基础.

2012 年, Almeida 课题组 ${ }^{[38]}$ 研究了石蒜碱衍生物对 抗寄生虫活性的作用, 将石蒜碱的 $\mathrm{C}(1), \mathrm{C}(2)$ 位置的羟 基进行修饰得到 6 种石蒜碱的衍生物 110 115, 其抗阴<smiles>O[C@H]1C=C2CCN3Cc4cc5c(cc4[C@H](C23)[C@H]1O)OCO5</smiles>

Lycorine

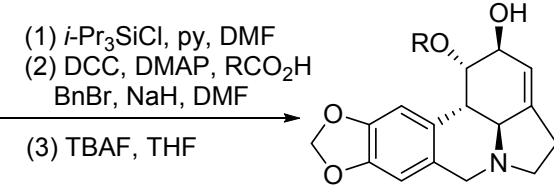

$89 \sim 96$

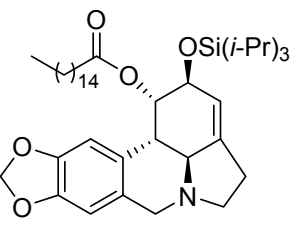

$8956 \%$

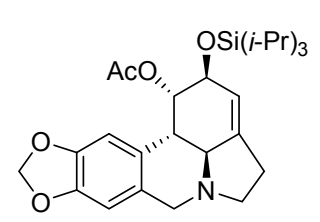

90 63\%

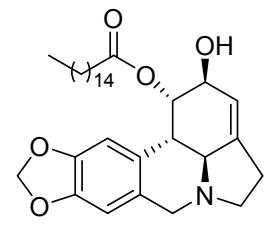

$9149 \%$<smiles>CC(=O)O[C@H]1[C@H](O)C=C2CCN3Cc4cc5c(cc4[C@H]2[C@H]13)OCO5</smiles>

$9251 \%$

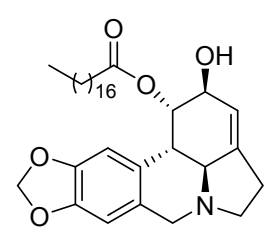

$9353 \%$<smiles>OC1C=C2CCN3Cc4cc5c(cc4[C@H](C23)[C@H]1O)OCO5</smiles>

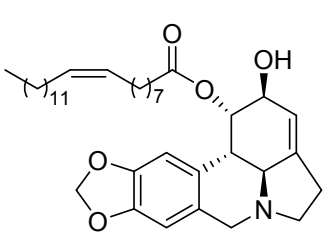

$94 \quad 41 \%$<smiles>CCC=CCCC(=O)O[C@H]1[C@H](O)C=C2CCN3Cc4cc5c(cc4[C@H]2[C@H]13)OCO5</smiles>

$95 \quad 51 \%$<smiles>O[C@H]1C=C2CCN3Cc4cc5c(cc4[C@@H]([C@H]1OCc1ccccc1)[C@H]23)OCO5</smiles>

$9630 \%$

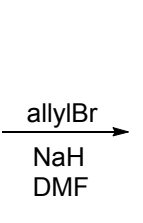<smiles>C=CCO[C@H]1C=C2CCN3Cc4cc5c(cc4[C@H]([C@H]1OCC=C)[C@H]23)OCO5</smiles>

$9735 \%$

图 4 Kornienko 及合作者合成的石蒜碱衍生物

Figure 4 Lycorine derivatives synthesized by Kornienko and co-workers 
<smiles>CCOC1C=C2CCN3Cc4cc5c(cc4[C@@H](C1OCC)[C@H]23)OCO5</smiles>

98<smiles>C#CCCO[C@H]1C=C2CCN3Cc4cc5c(cc4[C@H]([C@H]1OCC#C)[C@H]23)OCO5</smiles>

102<smiles></smiles>

106<smiles>CCCO[C@H]1C=C2CCN3Cc4cc5c(cc4[C@@H]([C@@H]1OCCC)[C@H]23)OCO5</smiles>

99<smiles>C#CCO[C@H]1C=C2CCN3Cc4cc5c(cc4[C@H]([C@H]1O)[C@H]23)OCO5</smiles>

103<smiles>O[C@H]1C=C2CCN3Cc4cc5c(cc4[C@@H]([C@@H]1OCC1CCC1)[C@H]23)OCO5</smiles>

107<smiles>C1=C2CCN3CCC(C(OC/C=C/c4ccccc4)[C@@H]1OC/C=C/c1ccccc1)[C@@H]23</smiles>

100<smiles>CC(C)=CCO[C@H]1C=C2CCN3Cc4cc5c(cc4[C@@H]([C@@H]1OCC=C(C)C)[C@H]23)OCO5</smiles>

104<smiles>COC1C(O[InH])C=C2CCN3Cc4cc5c(cc4[C@@H]1C23)OCO5</smiles>

108<smiles>O[C@H]1C=C2CCN3Cc4cc5c(cc4[C@@H]([C@@H]1OCC=Cc1ccccc1)[C@H]23)OCO5</smiles>

101<smiles></smiles>

105<smiles>CO[C@@H]1[C@H](O)C=C2CCN3Cc4cc5c(cc4[C@@H]1C23)OCO5</smiles>

109

图 5 Kornienko 报道的石蒜碱 C(1) 和 C(2)位置的双醚化反应产物

Figure 5 Double etherfication products at $\mathrm{C}(1)$ and $\mathrm{C}(2)$ positon of lycorine reported by Kornienko

道毛滴虫活性都强于石蒜碱, 其中 C(2)位置以月桂酰基 修饰的石蒜碱衍生物 111 抗阴道毛滴虫活性最好, 该工 作为进一步的机制研究提供了基础(图 6).

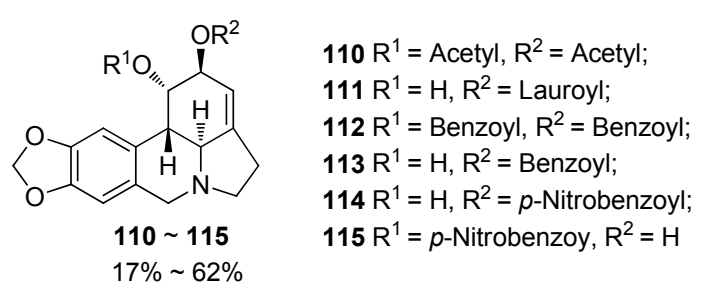

图 6 Almeida 课题组合成的石蒜碱衍生物

Figure 6 Lycorine derivatives synthesized by Almeida group

2009 年, Evidente 课题组 ${ }^{[39]}$ 合成 22 个石蒜碱衍生 物, 选取六种癌症细胞株并对其进行体外抗肿瘤活性测 试,虽然修饰的产物大部分的抗肿瘤活性都不如石蒜碱, 但其修饰方法及研究策略为抗肿瘤药物的发展做出了 贡献.

2010 年, Bastida 课题组 ${ }^{[40]}$ 将石蒜碱 C(2) 位置的着基 用叔丁基二甲基氯硅烷保护, 然后对 $\mathrm{C}(1)$ 位置的羟基进 行修饰, 所合成的新化合物对乙酰胆碱酶具有抑制作 用, 为推动治疗老年痴呆的新药研究提供了理论依据.

2008 年, Takayama 课题组 ${ }^{[41]}$ 从石蒜植物中提取了 新石蒜碱衍生物 LT1, 发现其具有良好的抗㾏原虫活性, 并对石赫碱进行类似修饰，合成了系列石赫碱类似物 116 和 117. 对其进行抗疮原虫活性测试, 其 $\mathrm{IC}_{50}$ 值显示
其具有良好的活性(Scheme 14). 同时，作者研究了石赫 碱衍生物对正常细胞毒性, 都显示出了满意的结果.

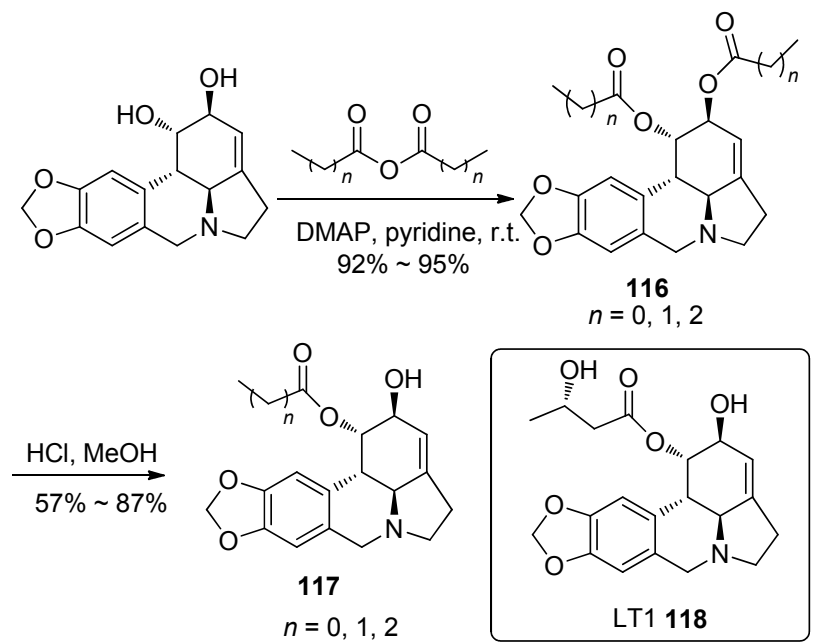

图式 14 Takayama 课题组合成的石蒜碱类似物 Scheme 14 Lycorine derivatives reported by Takayama group

\section{$2.2 \mathrm{C}(2)$ 位置的氧化}

对于石蒜碱 $\mathrm{C}(2)$ 位置的氧化, 最早在 1983 年, Evidente 等 ${ }^{[42]}$ 利用磷酸和 DCC 在干燥的 DMSO 溶解中反 应来制备. 最近通常采用琼斯试剂作为氧化剂, 但是这 两种方法的产率都很低. 2011 年, Kornienko 等 ${ }^{[36]}$ 选择利 用戴 斯-马丁试剂作为氧化剂，能够以 $66 \%$ 的产率得到 氧化产物. 2014 年, 尹正课题组 ${ }^{[34]}$ 用斯文氧化的条件获 得 $86 \%$ 的产率. 将 $\mathrm{C}(2$ )差弪基氧化成羰基能够增加抗西尼 
罗河病毒以及登革热病毒活性, 并且降低细胞毒活性. 以此化合物作为初始反应物对 $C(1)$ 进行修饰, 得到化合 物 120 125, 其活性都有满意的结果(Scheme 15).

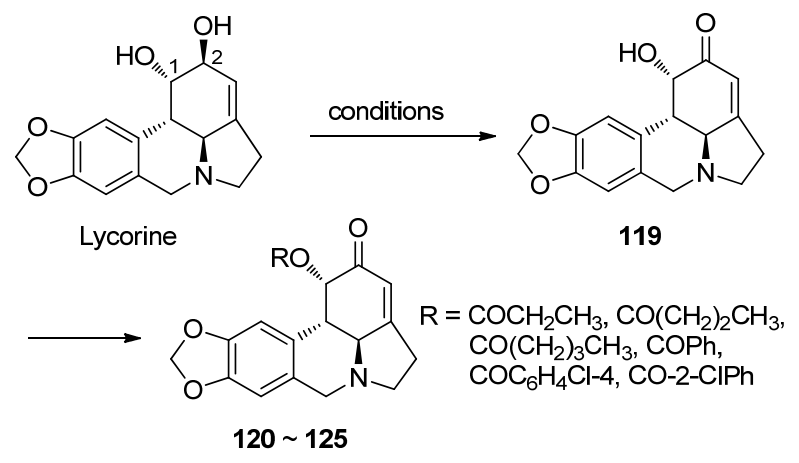

图式 15 尹正课题组报道了对石蒜碱 C(2)位置的氧化后对 $\mathrm{C}(1)$ 位置的酰化修饰

Scheme 15 Synthesis of lycorine derivatives with oxidation at $\mathrm{C}(2)$ position and then with acylation modifications at the $\mathrm{C}(1)$ position reported by Zheng Yin

\section{$2.3 \mathrm{C}(7)$ 位置的氧化和 D 环双键的还原}

2002 年, Lee 课题组 ${ }^{[43]}$ 利用醋酸碘苯氧化石蒜碱的 $\mathrm{C}(7)$ 位置, 以 $78 \%$ 的产率获得了 $\mathrm{C}(7)$ 位置氧化的石蒜碱 的衍生物 126, 同时以 $5 \%$ 的产率得到副产物 128 . 而 2011 年, Kornienko 课题组 ${ }^{[36]}$ 利用 $\mathrm{OsO}_{4} / \mathrm{NMO}$ 氧化得到 $53 \% \mathrm{C}(7)$ 位置氧化的石蒜碱产物 $\mathbf{1 2 7}$, 副产物 128 的产 率为 $13 \%$. 对以上的结构修饰做了构效关系研究, C(7) 位置的氧化不利于抗肿瘤活性的提高(Scheme 16).

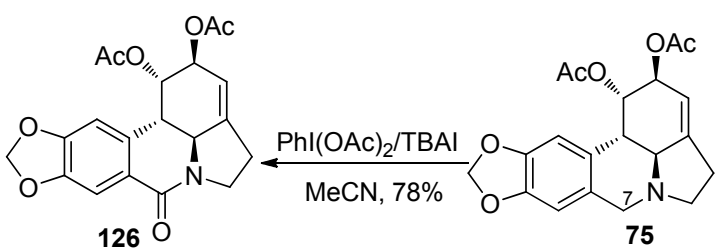

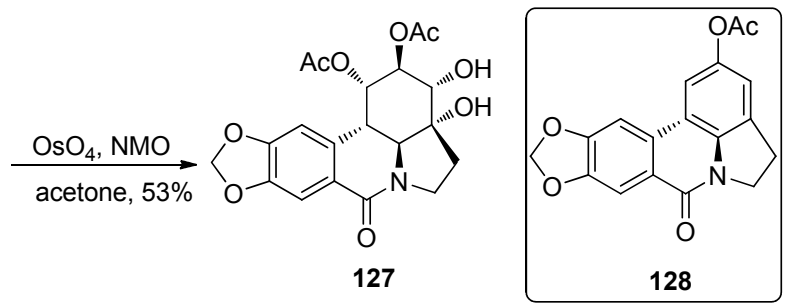

图式 16 Lee 课题组和 Kornienko 课题组报道的对石蒜碱 C(7) 位置进行结构修饰

Scheme 16 The modification at C(7) position of lycorine reported by Lee and Kornienko group

另外, D环双键的还原还可以在 $10 \% \mathrm{Pd} / \mathrm{C}$ 条件下还 原得到的, 所得产物 129 的抗㾏原虫活性降低(Scheme $17)^{[44]}$.

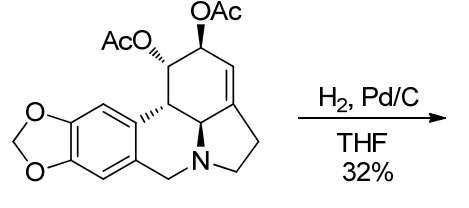

75<smiles>CC(=O)Oc1cc2c(cc1OC(C)=O)[C@H]1C(OC(C)=O)[C@H](OC(C)=O)C[C@H]3CCN(C2)[C@@H]31</smiles>

129
图式 17 石蒜碱的 D 环双键还原

Scheme 17 Reduction of double bond at D ring of lycorine

\section{$2.4 E$ 环开环和 $D$ 环的芳香化}

2007 年以前, 科研工作者们研究发现石蒜碱的优 良的生物活性, D 环起了决定作用, 但是科学家们并没 有证明石蒜碱的 E 环对活性有哪些影响, 2007 年, Lin 课 题组 ${ }^{[45]}$ 从文殊兰中提取的石蒜碱为反应物, 将其双乙 酰化, 然后在乙腈溶剂中与各种卤代烷反应生成季铵 盐. 首先在 $\mathrm{BuOK} / \mathrm{BuOH}$ 条件下消除双乙酰基将 $\mathrm{D}$ 环芳 香化, 然后发生霍夫曼降解反应将 $\mathrm{E}$ 环开环, 得到化合 物 132 137. 所得到的化合物进行体外活性测试发现, $\mathrm{E}$ 环的开环并没有影响其活性, 但可能是因为改变了溶 解性而增加了其活性. 该工作研究结果表明: D 环的芳 香化对乙酰胆碱酯酶的抑制活性增强(Scheme 18).

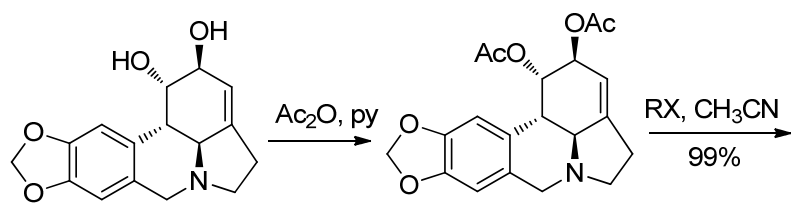

75

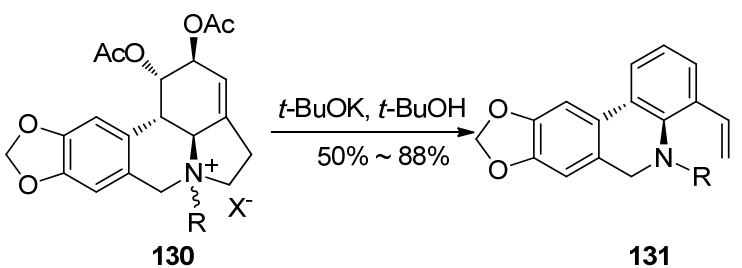

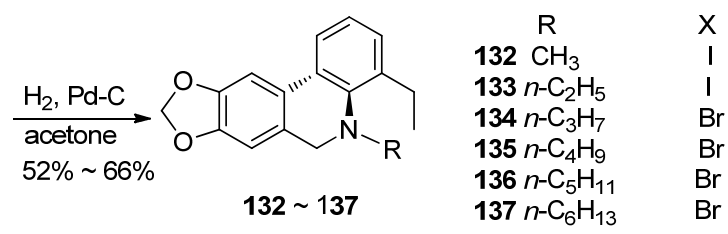

图式 18 Lin 课题组将石蒜碱 $\mathrm{E}$ 环开环并将 D 环芳香化 Scheme 18 The modification with open E ring and aromatization $\mathrm{D}$ ring of lycorine by Lin group

上述方法将 $\mathrm{E}$ 环开环，但是却改变了 $\mathrm{D}$ 环，使其发 生了芳香化. 如果用以下方法, 能够保留 $\mathrm{D}$ 环的原貌并 且将 E 环开环，但是体外活性研究发现，该方法所得到 的化合物的活性大大降低. 将双乙酰化的石蒜碱与三氯 乙基氯甲酸酯反应得到化合物 138 , 化合物 138 与 $\mathrm{NaOAc}$ 在 DMSO 中乙酰基对氯发生亲核取代反应生成 
化合物 139, 然后在锌粉催化下脱保护得到化合物 140, 再还原甲基化、脱乙酰化得到化合物 142 (Scheme 19).
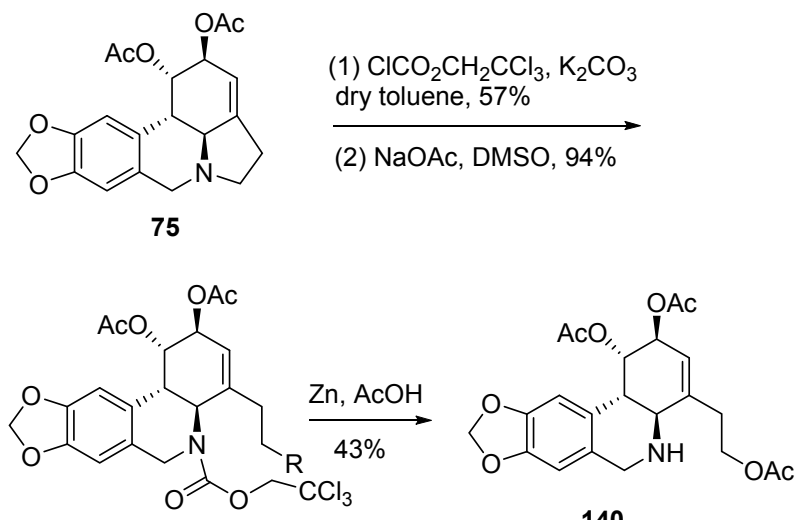

$138 \mathrm{R}=\mathrm{Cl}$

$139 \mathrm{R}=\mathrm{OAC}$

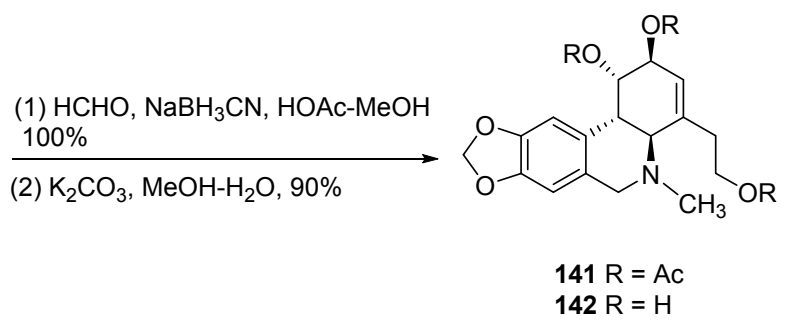

图式 19 对石蒜碱 $\mathrm{E}$ 环的开环

Scheme 19 Opening E ring of lycorine

2011 年, Kornienko 课题组在 D 环的芳香化上, 利用 氯化亚砜在吡啶中以 $92 \%$ 的产率得到 D 环芳香化产物 144 和 145, 对其进行抗肿瘤活性测试发现, D 环芳香化 后活性增强(Scheme 20) ${ }^{[36]}$.<smiles>C[13CH2][13C](=O)[O-]</smiles><smiles>CCN1C(=O)c2cc3c(cc2-c2cccc4c2N1CC4)OCO3</smiles>

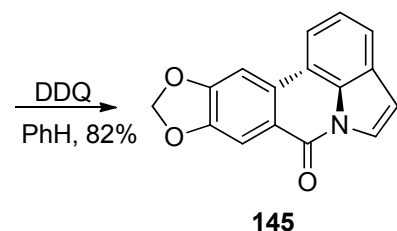

图式 20 Kornienko 课题组将石蒜碱的 D 环芳香化 Scheme 20 Aromatization D ring of lycorine by Kornienko group

\subsection{A 环的修饰}

2014 年, 尹正教授课题组 ${ }^{[34]}$ 对石蒜碱进行修饰, 研 究其抑制登革热病毒活性，探讨了 $\mathrm{A}$ 环的修饰，发现将 A 环 1,3-二氧戊环通过 $\mathrm{BBr}_{3}$ 催化下开环后得到化合物
146 和 147, 大大降低了对登革热病毒的活性, 对 1,3-二 氧戊环亚甲基进行修饰，经过还原、环化形成不同取代 基的 $\mathrm{A}$ 环产物 148, 该系列产物基本丧失对登革热病毒 的抑制活性. 以上研究结果表明 1,3-二氧戊环是活性所 需(Scheme 21).
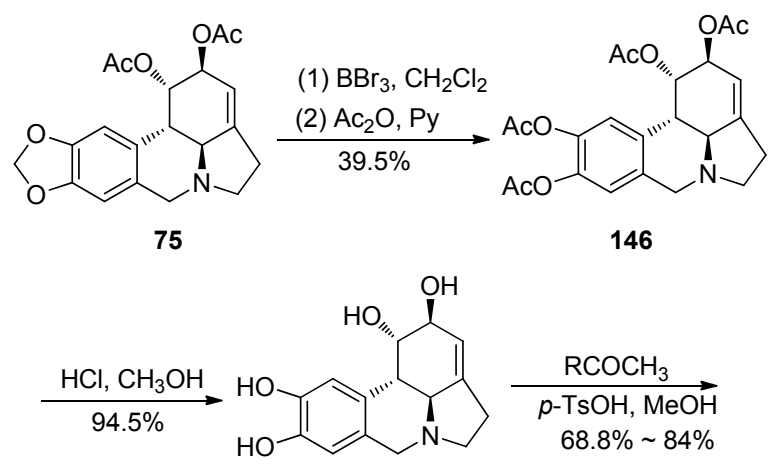

147

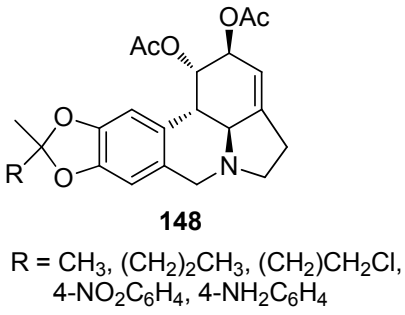

图式 21 尹正教授课题组对石蒜碱 A 环的修饰

Scheme 21 The modification of A ring of lycorine by Zheng Yin group

\section{2 总结与展望}

石蒜碱及其衍生物广泛的生物活性及药理作用引 起了有机合成及药物化学家的广泛关注. 近一个世纪以 来，对于石蒜碱的全合成已经取得了长足的进展，新的 催化剂及合成路线的开发使得合成效率有了较大幅度 的提升. 近年来，常常将不对称合成应用到石蒜碱的全 合成过程中，开辟了石蒜碱全合成的新方法，并为其他 生物碱的合成提供了新的途径. 对于石蒜碱的修饰，通 过取代基的改变和母核结构的改变以开发出活性更强 的石蒜碱衍生物仍然是药物化学家的重要课题, 特别是 近年来有关于石蒜碱及衍生物在抗肿瘤、抗病毒及其针 对老年痴呆病症的研究使得石蒜碱的研究越来越受到 重视. 因此，科学无止境，开发出更便捷、高效的合成石 蒜碱的方法及发现新型的石蒜碱衍生物的任务远未结 束，有待于进一步丰富和发展. 相信通过科学家们深入 的研究及不解的努力，石蒜碱类化合物必将被开发成为 新药带来重大的经济效益及巨大的学术价值.

\section{References}

[1] Huang, B. X.; Fu, S. G.; Zhu, P. L. Jiangxi Forestry Sci. Technol. 
2012, 40(1), 22 (in Chinese).

(黄宝祥, 符树根, 朱培林, 江西林业科技, 2012, 40(1), 22.)

[2] Cook, J. W.; Loudon, J. D. In The Alkaloids, Eds.: Manske, R. H. F.; Holmes, H. L., Academic Press, New York, 1952, Vol. II, pp. $331 \sim 352$.

[3] Kondo, H.; Uyeo, S. Chem. Ber. 1935, 68, 1756.

[4] Nakagawa, Y.; Uyeo, S. J. Chem. Soc. 1959, 3736.

[5] Lamoral-Theys, D.; Decaestecker, C.; Mathieu, V.; Dubois, J.; Kornienko, A.; Kiss, R.; Evidente, A.; Potter, L. Mini-Rev. Med. Chem. 2010, 10, 41.

[6] Cedron, J. C.; Gutierrez, D.; Flores. N.; Ravelo, A. G.; EstevezBraun, A. Bioorg. Med. Chem. 2010, 18, 4694.

[7] Toriizuka, Y.; Kinoshita, E.; Kogure, N.; Kitajima, M.; Ishiyama, A.; Otoguro, K.; Yamada, H.; Omura, S.; Takayama, H. Bioorg. Med. Chem. 2008, 16, 10182.

[8] Citoglu, G.; Tanker, M.; Gumusel, B. Phytother. Res. 1998, 12, 205.

[9] Tanker, M.; Citoglu, G.; Gumuhel, B.; Hener, B. Int. J. Pharmacogn. 1996, 34, 194.

[10] Kretzing, S.; Abrahama, G.; Seiwert, B.; Ungemach, F. R.; Krugel, U.; Regenthal, R. Toxicon 2011, 57, 117.

[11] Hwang, Y. C.; Chu, P. L.; Yang, W.; Chen, W.; Yates, M. V. Antiviral Res. 2008, 77, 232.

[12] Li, S. Y.; Chen, C.; Zhang, H.; Guo, H.; Wang, H.; Wang, L.; Zhang, X.; Hua, S.; Yu, J.; Xiao, P.; Li, R.; Tan, X. Antiviral Res. 2005, 67, 18.

[13] Renard-Nozaki, J.; Kim, T.; Imakura, Y.; Kihara, M.; Kobayashi, S. Res. Virol. 1989, 140, 115.

[14] Zhou, M.; Deng, L.; Kashanchi, F.; Brady, J. N.; Shatkin, A. J.; Kumar, A. Proc. Natl. Acad. Sci. U. S. A. 2003, 100, 12666.

[15] Gabrielsen, B.; Monath, T. P.; Huggins, J. W.; Kefauver, D. F. J. Nat. Prod. 1992, 55, 1569.

[16] Liu, J.; Li, Y.; Tang, L. J; Zhang, G. P.; Hu, W. X. Biomed. Pharmacother. 2007, 61, 229.

[17] Min, B. S.; Gao, J. J.; Nakamura, N.; Kim, Y. H.; Hattori, M. Chem. Pharm. Bull. 2001, 49, 1217.

[18] Cao, Z.; Yu, D.; Fu, S.; Zhang, G.; Pan, Y.; Bao, M,; Tu, J.; Shang, B.; Guo, P.; Yang, P.; Zhou, Q. Toxicol. Lett. 2013, 218, 174.

[19] Chapsal, B. D.; Ojima, I. Org. Lett. 2006, 8, 1395.

[20] Fujioka, H.; Murai, K.; Ohba, Y.; Hirose, H.; Kita, Y. Chem. Commun. 2006, 832;

[21] Dong, L.; Xu, Y. J.; Cun, L. F.; Cui, X.; Jiang, Y. Z.; Gong, L. Z. Org. Lett. 2005, 7, 4285;

[22] Banwell, M. G.; Harvey, J. E.; Hockless, D. C. R. J. Org. Chem. 2000, 65, 4241.

[23] Tsuda, Y.; Sano, T.; Taga, J.; Isobe, K.; Toda, J.; Irie, H.; Tanaka, H.; Takagi, S.; Yamaki, M.; Murata, M. J. Chem. Soc., Chem. Commun. 1975, 933.
[24] Trossell, K. Tetrahedron Lett. 1974, 623.

[25] Umezawa, B.; Hoshino, O.; Sawaki, S.; Sashida, H.; Mori, K.; Hamada, Y.; Kotera, K.; Iitaka, Y. Tetrahedron 1984, 40, 1783.

[26] Møller, O.; Steinberg, E. M.; Torssell, K. Acta Chem. Scand. Sect. B 1978, 32, 98.

[27] Yamada, K.; Yamashita, M.; Sumiyoshi, T.; Ni-Shimura, K.; Tomioka, K. Org. Lett. 2009, 11, 1631.

[28] Magnus, P.; Sebhat, I. K. J. Am. Chem. Soc. 1998, 120, 5341.

[29] Sun, Z. W.; Zhou, M. T.; Li, X.; Meng, X. L.; Peng, F. Z.; Zhang, H. B.; Shao, Z. H. Chem. Eur. J. 2014, 20, 6112.

[30] Schultz, A. G.; Holoboski, M. A.; Smyth, M. S. J. Am. Chem. Soc. 1996, 118, 6210 .

[31] Pozsgay, V.; Ohgi, T.; Hecht, S. M. J. Org. Chem. 1981, 46, 3763.

[32] Boeckman, R. J.; Goldstein, S. W.; Walters, M. A. J. Am. Chem. Soc. 1988, 110, 8250 .

[33] Elgorash, E. E.; Stafford, G. I.; Van Staden, J. Planta Med. 2004, $70(3), 260$

[34] Wang, P.; Li, L. F.; Wang, Q. Y.; Shang, L. Q.; Shi, P. Y.; Yin, Z. ChemMedChem 2014, 9, 1522.

[35] Tan, C. X.; Schrader, K. K.; Mizuno, C. S.; Rimando, A. M. J. Agric. Food Chem. 2011, 59, 5977.

[36] Evdokimov, N. M.; Theys, D. L.; Mathieu, V.; Andolfi, A.; Frolova, L. V.; Pelly, S. C.; Otterlo, W.; Magedov, I. V.; Kiss, R.; Evidente, A.; Kornienko, A. Bioorg. Med. Chem. 2011, 19, 7252.

[37] Dasari, R.; Banuls, L. M.; Masi, M.; Pelly, S. C.; Mathieu, V.; Green, I. R.; Otterlo, W. A. L.; Evidente, A.; Kiss, R.; Kornienko, A. Bioorg. Med. Chem. Lett. 2014, 24, 923.

[38] Giordani, R. B.; Junior, C. O. R.; Andrade, J. P.; Bastida, J.; Zuanazzi, J. A.; Tasca, T.; Almeida, M. V.; Chem. Biol. Drug Des. 2012, 80, 129.

[39] Lamora-Theys, D.; Andolfi, A.; Goietsenoven, G. V.; Cimmino, A.; Calve, B. L.; Wauthoz, N.; Megalizzi, V.; Gras, T.; Bruyere, C.; Dubois, J.; Mathieu, V.; Kornienko, A.; Kiss, R.; Evidente, A. J. Med. Chem. 2009, 52, 6244.

[40] Munulty, J.; Nair, J.; Little, J.; Brennan, J.; Bastida, J. Bioorg. Med. Chem. Lett. 2010, 20, 5290.

[41] Toriizuka, Y.; Kinoshita, E.; Kogure, N.; Kitajima, M.; Ishiyama, A.; Otoguro, K.; Yamada, H.; Omura, S.; Takayama, H. Bioorg. Med. Chem. 2008, 16, 10182.

[42] Evidente, A.; Cicala, M. R.; Randazzo, G.; Riccio, R.; Calabrese, G.; Liso, R.; Arrigoni, O. Phytochemisry 1983, 22, 2193.

[43] Huang, W. J.; Singh, O. V.; Chen, C. H.; Chiou, S. Y.; Lee, S. S. Helv. Chim. Acta 2002, 85, 1069.

[44] Cedron, J. C.; Gutierrez, D.; Plores, N.; Ravelo, A. C.; Estevez, B. A. Bioorg. Med. Chem. 2010, 18, 4694.

[45] Lee, S. S.; Venkatesham, U.; Rao, C. P. R.; Lam, S. H.; Lin, J. H. Bioorg. Med. Chem. 2007, 151034. 